\title{
Thyroiditis and SARS-CoV-2 pandemic: a review
}

\author{
Philippe Caron $\mathbb{D}^{1}$
}

Received: 4 January 2021 / Accepted: 10 March 2021 / Published online: 27 March 2021

(c) The Author(s), under exclusive licence to Springer Science+Business Media, LLC, part of Springer Nature 2021

\begin{abstract}
The world is dealing with the Covid-19 pandemic due to the coronavirus SARS-CoV-2. Amongst the extra-pulmonary manifestations presented by Covid-19 patients, thyroiditis form part of the spectrum of visceral involvement linked to SARS-CoV-2. In this review, we will describe the various documented clinical forms of thyroiditis (inflammatory thyroiditis, subacute or de Quervain's thyroiditis, chronic lymphocytic thyroiditis or Hashimoto's disease, painless (silent) postpartum thyroiditis) to facilitate their diagnosis in more or less symptomatic Covid-19 patients and to provide guidance for patient treatment.
\end{abstract}

Keywords Inflammatory thyroiditis • Destructive thyroiditis • Painful subacute thyroiditis $\cdot$ Painless postpartum thyroiditis · Hashimoto's thyroiditis $\cdot$ Thyroxine thyrotoxicosis

\section{Introduction}

Since late 2019, the world has been dealing with the pandemic due to SARS-CoV-2, a coronavirus which on the one hand involves mild flu-like or pauci-symptomatic forms in most young patients, but also multi-organ dysfunction (acute respiratory distress syndrome, septic shock, kidney failure) in other patients. This generally affects elderly patients and the prognosis depends on their degree of lung damage, which is sometimes severe. Extra-pulmonary manifestations may include endocrine forms involving pancreatic, pituitary, gonadal and finally thyroid disorders. Whereas the diagnosis of autoimmune hyperthyroidism or Graves' disease is rare, recent literature reports a more or less significant frequency of subacute or chronic thyroiditis in Covid-19 patients.

Characterising the clinical and hormonal features of thyroiditis seems necessary in patients with a mild or severe Covid-19 infection in order to make the diagnosis and analyse the physio-pathological mechanisms as these generally guide the treatment of these patients.

Philippe Caron

caron.p@chu-toulouse.fr

1 Department of Endocrinology and Metabolic diseases, Cardiovascular and Metabolic Unit CHU-Larrey, Toulouse, France
In this article, we will review the epidemiological, clinical, biochemical, radiographic and physio-pathological data on the various clinical forms of thyroiditis reported to date in patients with an infection due to SARS-CoV-2.

\section{Inflammatory or destructive thyroiditis}

In patients with more severe forms of Covid-19, epidemiological studies indicate an increased prevalence of thyrotoxicosis secondary to destructive or inflammatory thyroiditis $[1,2]$. In a retrospective study from Italy, $20.2 \%$ of patients hospitalised for a severe form of Covid-19 presented with clinical (10.8\%) or subclinical (9.4\%) thyrotoxicosis [1]. The prevalence of thyrotoxicosis is correlated with the increased concentration of interleukin-6, suggesting that thyroid lesions are due to inflammatory or destructive thyroiditis secondary to "the cytokine storm" observed in severe forms of SARSCoV-2 infection [3]. Muller et al. also report an increased prevalence of thyrotoxicosis in Covid-19 patients hospitalised in high intensity of care units for severe lung disease $(15 \%$, $n=85$ ) compared with that of Covid-19 patients hospitalised in low intensity of care units $(2 \%, n=41)$ and that of patients in high intensity of care units for severe non Covid-19 lung disease $(1 \%, n=78)$ [2]. These patients in Covid-19 high intensity of care units have an average age of $65 \pm 12$ years, are usually male (68\%) and have C-reactive protein (CRP) values (median 96, 51-177 $\mathrm{mg} / \mathrm{L}$ ) higher than patients in Covid-19 low intensity of care units (median 52, 22-103 mg/ L) and in non Covid-19 high intensity of care units (median 
$66,15-121 \mathrm{mg} / \mathrm{L}$ ), reflecting the significant inflammatory reaction associated with the SARS-CoV-2 infection. Therefore, the significant increase of pro-inflammatory cytokines (interleukin-6, TNF- $\alpha$ ) can be responsible of destructive thyroiditis [4]. In this context, a post mortem examination of patients who died from a SARS-CoV-1 infection revealed significant apoptosis of follicular cells with exfoliation of epithelial cells in the thyroid follicles [5].

From a clinical point of view, none of the patients with destructive thyroiditis complained of pain in the neck. The frequent occurrence of lymphopenia $(50-85 \%)$ in these severe forms of Covid-19 explains the lack of cell and granulomatous reaction in the thyroid. The absence of infiltration by neutrophils and lymphocytes into the thyroidal interfollicular spaces might explain the lack of tension of the thyroid capsule and consequently the non-painful nature of the inflammatory thyroiditis or "painless or silent thyroiditis" in these patients. Furthermore, the patients presented with thromboembolic complications (16.1\%) and episodes of atrial fibrillation (32.3\%) which increased hospital stay and the risk of death.

From a functional point of view, apoptosis of the follicular cells causes the release of preformed hormones and thyrotoxicosis with free $\mathrm{T} 4$ concentration either increased or at the upper limit of normal, and a low TSH concentration, meaning that thyrotoxicosis is less severe than that observed in patients with subacute thyroiditis. The free T3 concentration is reduced, a frequent anomaly in Covid-19 patients [6-10], mainly associated with inhibition of the deiodinase activity described in the low T3 syndrome or "Euthyroid Sick Syndrome" [11] secondary to "the cytokine storm" associated with severe SARS-CoV-2 infection. This anomaly of thyroid function during severe forms of Covid19 infection therefore produces T4-thyrotoxicosis [12], explaining the frequent absence of clinical symptoms of thyrotoxicosis in these patients. This T4-thyrotoxicosis associated with destructive thyroiditis is transient: after 55 days' follow-up, Muller et al. report that euthyroid conditions were restored in $75 \%$ of patients (6/8) with $25 \%$ $(2 / 8)$ of them developing hypothyroidism [2].

In the majority of the patients, the antithyroid antibodies (anti-thyroglobulin and anti-thyroid peroxidase) are negative ruling out autoimmune thyroiditis [1]. The thyroid ultrasound is either normal or reveals inflammatory signs (hypo-echogenic and heterogenous nature).

To summarise, during severe forms of SARS-CoV-2 infection, "the cytokine storm" can be associated with inflammatory or destructive thyroiditis with T4thyrotoxicosis. In patients with severe form of Covid-19 with thromboembolic complications and episodes of atrial fibrillation the treatment (heparin, glucocorticoids) may modify the parameters of the thyroid function [13] and make the diagnosis of thyrotoxicosis more difficult.
Monitoring will identify the onset of hypothyroidism in rare patients and verify the restoration of euthyroid conditions in the majority of patients.

Although on 13 March 2020 the World Health Organisation did not recommend the systematic evaluation of thyroid function in patients with Covid-19, the prevalence of the inflammatory thyroiditis justifies performing a thyroid function evaluation in patients hospitalised in high intensity of care units, and identifying any anomaly of thyroid function tests makes necessary a referral to an endocrinologist.

\section{Subacute or de Quervain's thyroiditis}

Subacute or de Quervain's thyroiditis is a granulomatous thyroiditis, usually during or after a viral episode in genetically predisposed patients (HLA-Bw35, HLA-B67, HLA-Drw8) [14]. Since the initial observation of subacute thyroiditis in a Covid-19 patient [15], at the present time 22 patients have been reported in the literature either as a clinical case report [15-26] or in small series of patients [27, 28] (Table 1).

From a clinical point of view, subacute thyroiditis is more common in women than in men (18 women/4 men), patients with an average age of $39 \pm 11$ years (mean \pm standard deviation), during or after ( $21 \pm 11$ days) an episode of Covid-19 which is either asymptomatic (contact patient) or mild with few symptoms. Patients may present general and infectious signs (fever, myalgia, asthenia), signs of thyrotoxicosis (palpitations, hyperhidrosis, weight loss) and pain in the anterior cervical region irradiating to the jaw or ear region, to be differentiated from an episode of pharyngitis which is frequent in Covid-19 patients. For Ippolito's patient, neck pain was absent but the patient was under high doses of painkillers after back surgery, possibly masking local symptoms [16]. Palpation of the anterior cervical region may identify a normal thyroid gland $(50 \%)$ or a goitre (50\%) varying in size, sensitive or painful, but the absence of painful phenomena does not rule out the diagnosis.

Laboratory tests reveal an inflammatory syndrome with increased CRP, mild leukocytosis with neutrophilia or a normal blood count (without lymphopenia), a low TSH level with a more or less significant increase in free $\mathrm{T} 4$ and T3 concentrations resulting in clinical or subclinical thyrotoxicosis, and in most patients an absence of anti-thyroid peroxidase antibodies whereas anti-thyroglobulin antibodies may be present in rare patients. The neck ultrasound shows a more or less significant goitre, heterogenous with bilateral diffuse hypoechoic areas without hypervascularisation at colour doppler ultrasonography, and few cervical lymph nodes with normal morphology. Scintigraphy shows that fixation of the radioisotope within the thyroid gland is markedly reduced or absent. 


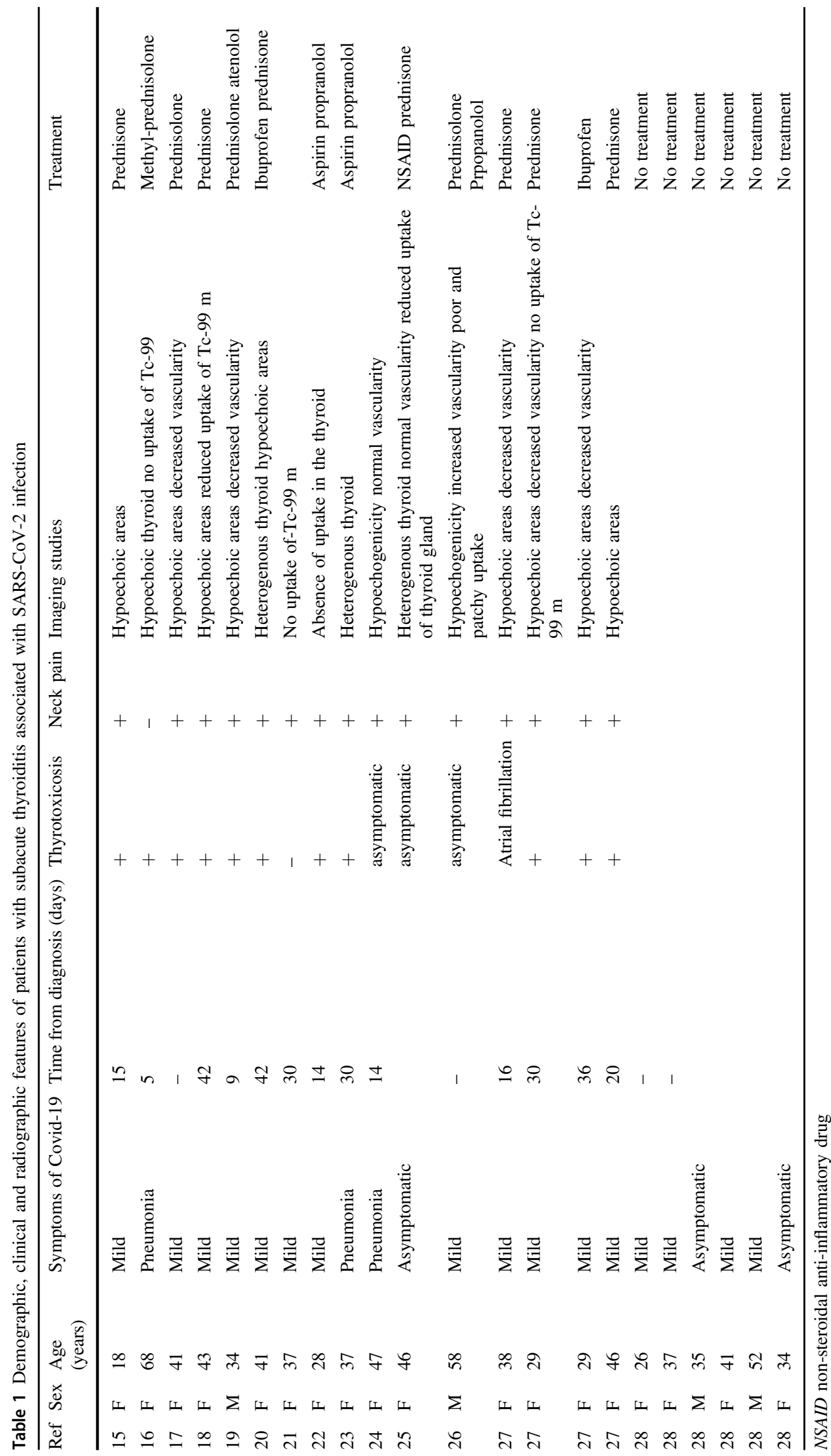


Patients were treated with $\beta$-blockers, aspirin, nonsteroidal anti-inflammatory drugs or cortico-steroids (rarely intravenously and more frequently orally) at low doses (prednisone $25-40 \mathrm{mg}$ ) gradually tapered over an average of 3 or 4 weeks. In most patients, despite a short follow-up ( $35 \pm 12$ days), a normal thyroid function is observed after a possible and transient episode of subclinical hypothyroidism. However, it is known that in longterm follow-up after viral thyroiditis there is a higher incidence of thyroid autoimmunity and hypothyroidism.

All the data are suggestive of subacute thyroiditis during or after a mild or asymptomatic episode of Covid-19. In most patients the absence of antithyroid antibodies rules out an episode of autoimmune thyroiditis. The clinical and paraclinical data are comparable to subacute thyroiditis observed during or after other viral infections caused by cytomegalovirus, rubella, Epstein Barr, enterovirus, adenovirus, coxsackies, measles, influenza [29].

From a physio-pathological point of view, SARS-CoV-1 and SARS-CoV-2 coronaviruses penetrate the organism from ear, nose and throat or pulmonary entry points. The presence of SARS-CoV-2 viral particles and mRNA in the serum of Covid-19 patients [30, 31] indicate episodes of viraemia. The angiotensin converting enzyme 2 (ACE2), a membranebound carboxypeptidase, with transmembrane protease serine 2 (TMPRSS2), is the receptor for SARS-CoV-2 coronavirus allowing it to penetrate the human cells $[32,33]$. ACE2 and TMPRSS2 are present in the thyroid follicular cells and their expression is greater than in the lung cells especially in women [34], which may explain the prevalence of subacute thyroiditis in women observed after an episode of infection, usually mild or moderate, by SARS-CoV-2.

From a practical point of view, subacute thyroiditis is rare during Covid-19 infections, but its recent description in Covid-19 patients means that de Quervain's thyroiditis must form part of the spectrum of extra-pulmonary symptoms of SARS-CoV-2 infection. A SARS-CoV-2 infection must be suspected and sought (reverse-transcription polymerase chain reaction test on ENT sample, serology, chest scan) during the etiological workup of de Quervain's thyroiditis, especially in women. Moreover current documented data shows the absence of recurrent subacute thyroiditis after treatment with low doses of corticosteroids for an episode of de Quervain's thyroiditis.

\section{Chronic lymphocytic thyroiditis}

Infection by SARS-CoV-2 coronavirus may lead to Th1/ Th17 hyperactivity which may trigger and maintain an inflammatory reaction [3] resulting in the onset of autoimmune disorders such as an antiphospholipid syndrome, autoimmune thrombocytopenia, autoimmune haemolytic anaemia, Guillain-Barre syndrome, and from a thyroid point of view chronic autoimmune thyroiditis or Hashimoto's thyroiditis [35] in Covid-19 patients. Currently the prevalence of Hashimoto's thyroiditis is rare after an episode of coronavirus infection: during convalescence, 3 months after an episode of infection with SARS-CoV-1, one patient (1.5\%) presented chronic lymphocytic thyroiditis responsible for primary thyroid insufficiency requiring definitive treatment with levothyroxine [36].

Thyroid hormones regulate the innate adaptive immune response by genomic and non-genomic mechanisms [37], and thyroid insufficiency increases susceptibility to infections. Indeed, during an infection with SARS-CoV-2, a 69-year-old female patient with sub-clinical autoimmune hypothyroidism presented a myxoedema coma complicated by cardiac arrest [38].

On the other hand, epidemiological studies show that patients with substituted hypothyroidism are not at risk of Covid-19 infection [39, 40], and that hypothyroidism treated with levothyroxine does not increase the risk of hospitalisation, stay in high intensity of care units and death compared with non-hypothyroid patients [41]. It means stressing the need to continue substitutive treatment with levothyroxine in patients with thyroid insufficiency secondary to autoimmune thyroiditis, taking precautions to guarantee supply particularly throughout the duration of any confinement.

Finally, after SARS-CoV-2 infection complicated by lymphopenia, one female patient presented a relapse of thyrotoxicosis associated with painless (silent) postpartum thyroiditis concurrent with a lymphocytic rebound, episode of thyroiditis with a spontaneously favourable outcome 7 months after birth [42]. This observation of painless (silent) postpartum thyroiditis following a SARS-CoV-2 infection could be compared with that described after a viral infection with the hepatitis $\mathrm{C}$ virus or viral-triggered postpartum thyroiditis [43]. Furthermore, fibrosis of the interfollicular connective tissue has been described in patients who died of the SARS-CoV-1 infection (5). Currently, no observation of Riedel thyroiditis or extensive fibrosis resulting in destruction of follicular cells and extending to surrounding organs has been reported after an infection with SARS-CoV-1 and SARS-CoV-2 coronavirus.

Author contributions P.C. had the idea of the review, performed the literature search and data analysis, and wrote the entire paper.

Funding No funds, grants, or other support was received by the author (P.C.) to assist with the preparation of this mini-review.

\section{Compliance with ethical standards}

Conflict of interest P.C. has no competing interests to declare that are relevant to the content of this article. 
Publisher's note Springer Nature remains neutral with regard to jurisdictional claims in published maps and institutional affiliations.

\section{References}

1. A. Lania, M.T. Sandri, M. Cellini, M. Mirani, E. Lavezzi, G. Mazziotti, On behalf of Humanitas COVID-19 Task force. Thyrotoxicosis in patients with Covid-19: the THYRCOV study. Eur. J. Endocrinol. 183, 381-387 (2020)

2. I. Muller, D. Cannavaro, D. Dazzi, D. Covelli, G. Mantovani, A. Muscatello et al. SARS-CoV-2-related atypical thyroiditis. Lancet Diabetes Endocrinol. 8, 739-741 (2020)

3. F. Coperchini, L. Chiovato, L. Croce, F. Magri, M. Rotondi, The cytokine storm in Covid-19: an overview of the involvement of the chemokine/chemokine-receptor system. Cytokine Growth Factor Rev. 53, 25-32 (2020)

4. W. Chen, Y. Tian, Z. Li, J. Zhu, T. Wei, J. Lei, Potential Interaction Between SARS-CoV-2 and Thyroid: a Review. Endocrinology 162(Mar 3), bqab004 (2021)

5. L. Wei, S. Sun, C.H. Xu et al. Pathology of the thyroid in severe acute respiratory syndrome. Hum. Pathol. 38, 95-102 (2007)

6. M. Chen, W. Zhou, W. Xu, Thyroid function analysis in 50 patients with COVID-19: a retrospective study. Thyroid (2020). https://doi.org/10.1089/thy.2020.0363

7. T. Chen, D. Wu, H. Chen, W. Yan, D. Yang, G. Chen, K. Ma, D. Xu, H. Yu, H. Wang, T. Wang, W. Guo, J. Chen, C. Ding, X. Zhang, J. Huang, M. Han, S. Li, X. Luo, J. Zhao, Q. Ning, Clinical characteristics of 113 deceased patients with coronavirus disease 2019: retrospective study. BMJ. (2020). https://doi.org/10.1136/bmj.m1091

8. T. Li, L. Wang, H. Wang, Y. Gao, X. Hu, X. Li, S. Zhang, Y. Xu, W. Wei, Characteristics of laboratory indexes in COVID-19 patients with non-severe symptoms in Hefei City, China: diagnostic value in organ injuries. Eur. J. Clin. Microbiol. Infect. Dis. 39, 2447-2455 (2020)

9. D. T. W. Lui, C. H. Lee, W. S. Chow, A. C. H. Lee, A. R. Tam, C. H. Y. Fong, C. Y. Law, E. K. H. Leung, K. K. W. To, K. C. B. Tan, Y. C. Woo, C. W. Lam, I. F. N. Hung, K. S. L. Lam, Thyroid Dysfunction in Relation to Immune Profile, Disease Status and Outcome in 191 Patients with COVID-19. J. Clin. Endocrinol. Metab, (2020). https://doi.org/10.1210/clinem/dgaa813

10. B. Khoo, T. Tan, S. A. Clarke, E. G. Mills, B. Patel, M. Modi, M. Phylactou, P. C. Eng, L. Thurston, E. C. Alexander, K. Meeran, A. N. Comninos, A. Abbara, W. S. Dhillo, Thyroid function before, during and after COVID-19. J. Clin. Endocrinol. Metab. (2020). https://doi.org/10.1210/clinem/dgaa830.

11. R. Zou, C. Wu, S. Zhang, G. Wang, Q. Zhang, B. Yu, Y. Wu, H. Dong, G. Wu, S. Wu, Y. Zhong, Euthyroid Sick Syndrome in Patients With COVID-19. Front. Endocrinol. (Lausanne). (2020). https://doi.org/10.3389/fendo.2020.566439

12. E. Fliers, A.C. Bianco, L. Langouche, A. Boelen, Thyroid function in critically ill patients. Lancet Diabetes Endocrinol. 3, 816-825 (2015)

13. J.R. Stockigt, C.F. Lim, Medications that distort in vitro tests of thyroid function, with particular reference to estimates of serum free thyroxine. Best. Pract. Res. Clin./. Endocrinol. Metab. 23, 753-767 (2009)

14. L.B. Sweeney, C. Stewart, D.Y. Gaitonde, Thyroiditis: an integrated approach. Am. Fam. Physician 90, 389-396 (2014)

15. A. Brancatella, D. Ricci, N. Viola, D. Sgro, F. Santini, F. Latrofa, Subacute thyroiditis after SARS-CoV-2 infection. J. Clin. Endocrinol. Metab. 105, 1-4 (2020)

16. S. Ippolito, F. Dentali, M.L. Tanda, SARS-CoV-2: a potential trigger for subacute thyroiditis? Insights from a case report. J. Endocrinol. Investig. 43, 1171-1172 (2020)
17. E. Asfuroglu Kalkan, I. Ates, A case of subacute thyroiditis associated with Covid-19 infection. J. Endocrinol. Investig. 43, 1173-1174 (2020)

18. R. M. Ruggeri, A. Campenni, M. Siracusa, G. Frazzetto, D. Gullo, Subacute thyroiditis in a patient infected with SARS-COV-2: an endocrine complication linked to the COVID-19 pandemic. Hormones (2020). https://doi.org/10.1007/s42000-020-00230-w

19. S. A. M. Mattar, S. J. Q. Koh, S. Rama Chandran, B. P. Z. Cherng, Subacute thyroiditis associated with COVID-19. BMJ Case Rep. (2020). https://doi.org/10.1136/bcr-2020-237336

20. A. Khatri, E. Charlap, A. Kim, Subacute thyroiditis from COVID19 infection: A case report and review of literature. Eur. Thyroid. J 9, 324-328 (2021). https://doi.org/10.1159/000511872

21. E. Campos-Barrera, T. Alvarez-Cisneros, M. Davalos-Fuentes, Subacute Thyroiditis Associated with COVID-19. Case Rep. Endocrinol. (2020). https://doi.org/10.1155/2020/8891539

22. R. Ruano, M. Zorzano-Martinez, A. Campos, F. Rius, M. Hernández, Subacute thyroiditis might be a complication triggered by SARS-CoV-2. Endocrinol. Diabetes Nutr. (2020). https://doi.org/ 10.1016/j.endinu.2020.09.002

23. W. H. Chong, B. Shkolnik, B. Saha, Subacute Thyroiditis in the Setting of Coronavirus Disease 2019. Am. J. Med. Sci. (2020). https://doi.org/10.1016/j.amjms.2020.09.011

24. M.D.J. San Juan, M.Q.V. Florencio, M.H. Joven, Subacute thyroiditis in a patient with coronavirus disease 2019. AACE Clin. Case Rep. 6, e361-e364 (2020)

25. M. C. Álvarez Martín, C. Del Peso Gilsanz, A. Hernández López, Subacute De Quervain thyroiditis after SARS-CoV-2 infection. Endocrinol. Diabetes Nutr. (2020). https://doi.org/10.1016/j. endinu.2020.10.003

26. U. Chakraborty, S. Ghosh, A. Chandra, A. K. Ray,, Subacute thyroiditis as a presenting manifestation of COVID-19: a report of an exceedingly rare clinical entity. BMJ Case Rep. (2020). https:// doi.org/10.1136/bcr-2020-239953

27. A. Brancatella, D. Ricci, D. Cappellani, N. Viola, D. Sgrò, F. Santini, F. Latrofa, Is Subacute Thyroiditis an Underestimated Manifestation of SARS-CoV-2 Infection? Insights From a Case Series. J. Clin. Endocrinol. Metab. (2020). https://doi.org/10.1210/ clinem/dgaa537

28. S. Sohrabpour, F. Heidari, E. Karimi, R. Ansari, A. Tajdini, F. Heidari, Subacute thyroiditis in COVID-19 patients. Eur. Thyroid. J. 9, 321-323 (2021). https://doi.org/10.1159/000511707

29. R. Desailloud, D. Hober, Viruses and thyroiditis: an update. Virol. J. 6, 5 (2009)

30. W. Wang, Y. Xu, R. Gao, R. Lu, K. Han, G. Wu, W. Tan, Detection of SARS-CoV-2 in Different Types of Clinical Specimens. JAMA 323, 1843-1844 (2020)

31. L. Chang, Y. Yan, L. Wang, Coronavirus disease 2019: coronaviruses and blood safety. Transfus. Med. Rev. 34, 75-80 (2020)

32. E. Lazartigues, M. M. F. Qadir, F. Mauvais-Jarvis. Endocrine significance of SARS-CoV-2's reliance on ACE2. Endocrinology. (2020). https://doi.org/10.1210/endocr/bqaa108

33. M. Rotondi, F. Coperchini, G. Ricci, M. Denegri, L. Croce, S. T. Ngnitejeu, L. Villani, F. Magri, F. Latrofa, L. Chiovato, Detection of SARS-COV-2 receptor ACE-2 mRNA in thyroid cells: a clue for COVID-19-related subacute thyroiditis. J. Endocrinol. Investig. (2020). https://doi.org/10.1007/s40618-020-01436-w

34. M.Y. Li, L. Li, Y. Zhang, X.S. Wang, Expression of the SARSCoV-2 cell receptor gene ACE2 in a wide variety of human tissues. Infect. Dis. Poverty 9, 45 (2020)

35. L. Y. Tee, S. Harjanto, B. H. Rosario, COVID-19 complicated by Hashimoto's thyroiditis. Singapore Med. J. (2020). https://doi.org/ 10.11622/smedj.2020106

36. M.K.-S. Leow, D.S.-K. Kwek, A.W.-K. Ng, K.-C. Ong, G.J.-L. Kaw, L.S.-U. Lee, Hypocortisolism in survivors of severe acute 
respiratory syndrome (SARS). Clin. Endocrinol. 63, 197-202 (2005)

37. P. De Vito, S. Incerpi, J.Z. Pedersen, P. Luly, F.B. Davis, P.J. Davis, Thyroid hormones as modulators of immune activities at the cellular level. Thyroid 21, 879-890 (2011)

38. N. M. Dixit, K. P. Truong, S. V. Rabadia, D. Li, P. K. Srivastava, T. Mosaferi, M. A. Calfon Press, I. Donangelo, T. Kelesidis, Sudden Cardiac Arrest in a Patient With Myxedema Coma and COVID-19. J. Endocr. Soc. (2020). https://doi.org/10.1210/jendso/bvaa130

39. P.K. Bhatraju, B.J. Ghassemieh, M. Nichols, R. Kim, K.R. Jerome, A.K. Nalla, A.L. Greninger, S. Pipavath, M.M. Wurfel, L. Evans, P. A. Kritek, T.E. West, A. Luks, A. Gerbino, C.R. Dale, J.D. Goldman, S. O'Mahony, C Mikacenic. Covid-19 in Critically Ill Patients in the Seattle Region. N. Engl. J. Med. 382, 2012-2022 (2020)
40. D. Dworakowska, A.B. Grossman, Thyroid disease in the time of COVID-19. Endocrine 68, 471-474 (2020)

41. M. van Gerwen, M. Alsen, C. Little, J. Barlow, L. Naymagon, D. Tremblay, C. F. Sinclair, E. Genden, Outcomes of Patients With Hypothyroidism and COVID-19: A Retrospective Cohort Study. Front. Endocrinol. (Lausanne). (2020). https://doi.org/10.3389/ fendo.2020.00565

42. S. Mizuno, H. Inaba, K. I. Kobayashi, K. Kubo, S. Ito, T. Hirobata, G. Inoue, T. Akamizu, N. Komiya, A case of postpartum thyroiditis following SARS-CoV-2 infection. Endocr. J. (2020). https://doi.org/10.1507/endocrj.EJ20-0553

43. I.S. Elefsiniotis, E. Vezali, K.D. Pantazis, G. Saroglou, Postpartum thyroiditis in women with chronic viral hepatitis. J. Clin. Virol. 41, 318-319 (2008) 